$12-1-1987$

\title{
Moment Analysis of the Cluster-Size-Distribution Approach to Scaling During Coagulation
}

Thomas W. Taylor

Cleveland State University, t.taylor@csuohio.edu

C. M. Sorensen

Follow this and additional works at: https://engagedscholarship.csuohio.edu/sciphysics_facpub

Part of the Physics Commons

How does access to this work benefit you? Let us know!

Publisher's Statement

Copyright 1987 American Physical Society. Available on publisher's site at http://pra.aps.org/ abstract/PRA/v36/i11/p5415_1.

\section{Original Citation}

Taylor, Thomas W. and C. M. Sorensen. "Moment Analysis of the Cluster-Size-Distribution Approach to Scaling During Coagulation." Physical Review A 36 (1987): 5415-5419.

\section{Repository Citation}

Taylor, Thomas W. and Sorensen, C. M., "Moment Analysis of the Cluster-Size-Distribution Approach to Scaling During Coagulation" (1987). Physics Faculty Publications. 22.

https://engagedscholarship.csuohio.edu/sciphysics_facpub/22

This Article is brought to you for free and open access by the Physics Department at EngagedScholarship@CSU. It has been accepted for inclusion in Physics Faculty Publications by an authorized administrator of

EngagedScholarship@CSU. For more information, please contact library.es@csuohio.edu. 


\title{
Moment analysis of the cluster-size-distribution approach to scaling during coagulation
}

\author{
T. W. Taylor \\ Department of Physics, Cleveland State University, Cleveland, Ohio 44115 \\ C. M. Sorensen \\ Department of Physics, Kansas State University, Manhattan, Kansas 66506
}

(Received 18 May 1987)

\begin{abstract}
We study the temporal approach of a cluster size distribution to its asymptotic scaling form. By enforcing consistency between the distribution's zeroth moment derived from both the Smoluchowski equation and the scaling distribution ansatz, we find values for the scaling exponents $w$ and $z$ in terms of the scaling exponent $\tau$ and the kernel homogeneity $\lambda$ which are not equivalent to their asymptotic, scaling forms. The predicted values do agree well, however, with intermediate time values found in simulations by Kang, Redner, Meakin, and Leyvraz [Phys Rev. A 33, 1171 (1986)]. By enforcing consistency between all moment orders, the asymptotic exponent values are found. These results imply the lowest-order moments approach their scaling values quickest.
\end{abstract}

\section{INTRODUCTION}

There has been considerable interest in recent years in aggregation processes because of the wide range of applications. ${ }^{1-3}$ The main thrust of recent work has been in irreversible aggregation and the scaling properties of the cluster size distribution. ${ }^{4,5}$ The Smoluchowski coagulation equation (SE) describes the time evolution of the cluster size distribution in the mean-field approximation and is the fundamental equation in this area of research. ${ }^{6}$ The SE equation is

$$
\dot{n}_{k}=\frac{1}{2} \sum_{(i+j=k)} K(i, j) n_{i} n_{j}-n_{k} \sum_{j=1}^{\infty} K(j, k) n_{j}
$$

In Eq. (1) $n_{k}$ is the concentration of clusters with $k$ monomers per cluster and $K(i, j)$ is the coagulation kernel and represents the rate coefficient for the clustering process between clusters of size $i$ and $j$.

One of the important findings in the study of the SE with a homogeneous coagulation kernel is that for times that are long compared to the characteristic coagulation time, the concentration or size distribution has the scaling form ${ }^{4}$

$$
n_{k}(t)=M_{1} k^{-2} \psi(k / s) .
$$

In Eq. (2) $M_{1}$ is the total number of monomers in the system and must remain constant, $\psi(x)$ is a timedependent scaling function whose form depends on the coagulation kernel, and $s$ is the cluster mean size. Using this form for the cluster size distribution, scaling exponents are defined and a relationship between them is found. Asymptotic values of the exponents have been found for certain forms of the coagulation kernel.

In an effort to corroborate these theoretical results, computer simulations of the cluster-cluster aggregation process were recently performed by Kang et al. ${ }^{7}$ They uncovered some surprising results. An intermediate time regime was found to exist where the scaling relation was obeyed by the exponents, but the values of the exponents were not the predicted asymptotic values. The exponents also changed in value with time, and the length of time required to reach asymptotic values depended on the form of the coagulation kernel. In an effort to understand the computer simulations, it became apparent that the long-time scaling solution to the SE, Eq. (2), was not the solution at these intermediate times. However, since the exponent relationship holds, it must be a good approximation.

Lee, ${ }^{8}$ in an earlier work, was able to study many of the features of coagulation processes using an approximation for the cluster size distribution. He analyzed the moments of the distribution rather than the distribution itself. Even though the moment equation carried less information than the equation for the size distribution, this procedure was effective because the gross features of the coagulation process are present.

In this paper we describe a procedure which uses the moment equations that lead to approximate exponent relations. These relations are valid whenever the scaling solution of the cluster size distribution is a good approximation and the exponents are weak functions of time. The key to this procedure is to demand consistency between the time evolution of the moments derived in two ways: one using the long-time scaling form of the cluster size distribution, Eq. (2), and the other using the SE, Eq. (1). If we enforce consistency only for the zeroth moment, the lowest-order description of the time evolution of the size distribution, we obtain additional exponent relations that agree with the results of Kang et al. However, if consistency is required for all the moments, then Eq. (2) is the exact solution to the SE and the previously determined asymptotic exponent relations are obtained.

We interpret this to mean that as the cluster size distribution evolves from some arbitrary shape to the asymptotic scaling form, the first distribution moment to achieve its asymptotic value is the zeroth moment. 
Thereafter, higher-order moments achieve their asymptotic values as well, leading to the exact scaling distribution; hence, the exact exponent relations in the infinite time limit.

\section{THEORY}

It is helpful at this time to discuss the properties of the coagulation kernel and the scaling form of the cluster size distribution.

There are three main kernels that appear in the literature. $^{1-3}$ These are the Brownian, product, and sum kernels. The Brownian kernel $K_{B}(i, j)=\left(i^{\beta}+j^{\beta}\right)\left(i^{-\beta}+j^{-\beta}\right)$ is used to describe the Brownian coagulation of clusters. The first term represents the geometrical collision cross section, and the second term represents the size dependence of the diffusion coefficients of the clusters. The product kernel $K_{p}(i, j)=i^{\beta} j^{\beta}$ has been used to describe branched polymerization. The sum kernel $K_{s}(i, j)=i^{\beta}$ $+j^{\beta}$ is used to describe the Brownian coagulation of clusters when the diffusion coefficients are independent of cluster size.

While the kernels above relate to real physical situations, the mathematical structure of the theory is better defined by more general properties of the kernels. Thus van Dongen and Ernst ${ }^{9}$ characterize the kernels by two exponents $\lambda$ and $\mu$, defined by

$$
\begin{aligned}
& K(a i, a j)=a^{\lambda} K(i, j), \\
& K(i, j) \sim i^{\mu} j^{v} \quad(j \gg i) .
\end{aligned}
$$

Equation (3) defines the homogeneity of the kernel, $\lambda=\mu+\nu$, and the exponent $\mu$ further divides the kernel into three separate classes. In class I, $\mu>0$; in class II, $\mu=0$; and in class III, $\mu<0$. For non-negative homogeneities $\lambda \geq 0$, the product, sum, and Brownian kernels are class I, II, and III, respectively. If $\lambda \geq 1$, mass is not conserved and the system gels. ${ }^{9}$

When the cluster size distribution has the scaling form, Eq. (2), the cluster mean size $s$ scales with time as $t^{z}$. The other scaling exponents are found when one considers the small- $x$ limit of $\psi(x)$.

For class I, $\psi(x) \sim x^{w / z}$ for $x<1$, where $w$ is a temporal scaling exponent. For class III, $\psi(x)$ $\sim \exp \left(-x^{-|\mu|}\right)$ for $x<1$. Class-II systems appear to depend on the details of the kernel. For our purposes we shall take $\psi(x) \sim x^{w / z}$ when $x<1$ for the sum kernel (class II), as this is consistent with the simulation result of Kang et al. To summarize, the kernels we will consider will yield the following $x<<1$ properties:

$$
\begin{aligned}
& \psi(x) \sim x^{w / z} \quad(\text { sum and product) } \\
& \psi(x) \sim \exp \left(-x^{-|\mu|}\right) \quad \text { (Brownian). }
\end{aligned}
$$

Application of Eq. (5a) to Eq. (2) yields for $x<1$

$$
n_{k}(t) \sim k^{-\tau} t^{-w},
$$

where $\tau$ is defined by the scaling relation

$$
w=z(2-\tau)
$$

and $\tau$ is constrained to $\tau<2$ by the conservation of mass.

In the following formulation we will constrain our- selves to nongelling, mass conserved systems so that $0 \leq \lambda<1$ and $\tau<2$. We also make the following three assumptions.

(i) The discrete form of the SE can be replaced by the continuous form.

(ii) The cluster size distribution has evolved sufficiently so that it may be approximated by

$$
n(v)=M_{1} v^{-2} \psi\left(v / t^{z}\right),
$$

where $v \sim k$ is the cluster volume.

(iii) The exponents $w, \tau$, and $z$ are at most weak functions of time.

The moments of the cluster size distribution are defined by

$$
M_{i}=\int_{0}^{\infty} v^{i} n(v) d v .
$$

Using the approximate scaling form (8) and making a change of variables, the moments can be written as

$$
M_{i}=M_{1} t^{z(i-1)} P_{i}
$$

where

$$
P_{i}=\int_{0}^{\infty} x^{i-2} \psi(x) d x
$$

The integral (11) may diverge at either the upper or lower limits. To ensure that it does not, we will consider separately the two different small- $x$ limits, Eqs. (5), of the scaling function. For the product and sum kernels the small- $x$ limit of the scaling function is [Eq. (5a)]

$$
\psi(x) \sim x^{w / z},
$$

and the large- $x$ limit must be $\psi(x)=0$. This means that the integral (11) is either finite or dominated by the lower end of the scaling function.

Substitution of Eq. (5) into Eq. (11) and using Eq. (7), one finds

$$
P_{i} \sim \int_{0} x^{i-\tau} d x
$$

This integral is finite for $\tau<i+1$; however, it diverges at the lower limit for $\tau \geq i+1$. To resolve this problem we revert back to volume space and note that the lower limit is now $v_{1}$, the volume of a monomer. Therefore, the integral is finite and we obtain

$$
P_{i} \sim t^{-z(i-1) w} \int_{v_{1}} v^{i-\tau} d v, \quad \tau \geq i+1 .
$$

Using Eqs. (10) and (13) the time dependence of the moments for the product and sum kernels are

$$
\begin{aligned}
& M_{i} \sim t^{z(i-1)}, \quad \tau<i+1 \\
& M_{i} \sim t^{-w}, \quad \tau \geq i+1 .
\end{aligned}
$$

Next we consider the Brownian kernel. In view of Eq. (5b), the integral (9) is finite for all values of $i$ and the time dependence of the moment is given by Eq. (14a). Because of this, we will concern ourselves only with the product and sum kernels for the rest of this section, remembering that the results for the Brownian kernel are the same as for the product and sum kernels when $\tau<1$ 
The next step is the central one in this approach to the exponent relations. We now require the rate at which the moments change as given in Eqs. (14) equal the rate at which they are required to change using the SE. Multiplying the SE in Eq. (1) by $k^{i}$, summing over all $k$, and converting to the continuous form, gives

$$
\dot{M}_{i}=\int_{0}^{\infty} d v \int_{0}^{\infty} d u\left[(v+u)^{i}-v^{i}-u^{i}\right] K(u, v) n(v) n(u)
$$

Using Eq. (8) and changing variables, Eq. (15) becomes

$$
\dot{M}_{i}=M_{1}^{2} t^{z(i+\lambda-2)} I_{i}(K),
$$

where

$$
\begin{gathered}
I_{i}(K)=\int_{0}^{\infty} d x \int_{0}^{\infty} d y\left[(x+y)^{i}-x^{i}-y^{i}\right](x y)^{-2} \\
\times K(x, y) \psi(x) \psi(y) .
\end{gathered}
$$

Here again we must make sure that Eq. (17) does not diverge at the lower limit. It is at this point that we use the assumption that the scaling form of the cluster size distribution is only an approximation. If it were exact, we would require that Eq. (16) be equivalent to the time derivatives of Eq. (14) for all $i$. Since it is approximate, we only require that they hold for a single $i$. We choose $i=0$ because it is the lowest-order description of the evolution of the size distribution. Also consideration of the zeroth moment causes divergences in Eq. (17) at the lower limit, and it is these divergences that yield the additional exponent relations. Because Eq. (16) is also kernel dependent, we must analyze the two kernels separately. Since the procedure is the same for both kernels, we will describe the procedure for the product kernel and report the exponent relations for all three kernels.

Substitution of the product kernel into Eq. (17) with $i=0$, we obtain

$$
I_{0}\left(K_{p}\right)=-\int_{0}^{\infty} d x \int_{0}^{\infty} d y(x y)^{\beta-2} \psi(x) \psi(y) .
$$

Because of a possible divergence of Eq. (18) at the lower limit, the small-x limit for the scaling functions, Eq. (5a), is used to obtain

$$
I_{0}\left(K_{p}\right)=-\int_{0} d x x^{\beta-2+w / z} \int_{0} d y y^{\beta-2+w / z} .
$$

This integral is finite for $\beta-2+w / z>-1$ or, using Eq. (17) and $\lambda=2 \beta, \tau<1+\lambda / 2$. However, if $\tau \geq 1+\lambda / 2$, Eq. (19) diverges at the lower limit and we must convert back to volume space, where the integrals are finite. This gives
TABLE I. Scaling exponents $w$ and $z$ for various kernels of homogeneity $\lambda$ and various ranges of $\tau$, derived under the assumption that only the zeroth moment of the cluster size distribution accurately reflects the scaling form.

\begin{tabular}{lccc}
\hline \hline \multicolumn{1}{c}{ Kernel } & $\tau$ range & $w$ & $z$ \\
\hline Product & $\tau<1$ & $\frac{2-\tau}{1-\lambda}$ & $\frac{1}{1-\lambda}$ \\
Product & $1 \leq \tau<1+\frac{\lambda}{2}$ & $\frac{2-\tau}{\tau-\lambda}$ & $\frac{1}{\tau-\lambda}$ \\
Product & $\tau \geq 1+\lambda / 2$ & 1 & $\frac{2}{2-\tau}$ \\
Sum & $\tau<1+\lambda$ & $\frac{2-\tau}{1-\lambda}$ & $\frac{1}{1-\lambda}$ \\
Sum & $\tau \geq 1+\lambda$ & $\frac{2-\tau}{1}$ & $\frac{1}{1-\tau}$ \\
Brownian & all $\tau$ & $\frac{1-\lambda}{1-\lambda}$ \\
\hline \hline
\end{tabular}

$I_{0}\left(K_{p}\right) \sim-t^{-2 z(\beta-1+w / z)} \int_{v_{1}} d v \int_{u_{1}} d u(u v)^{\beta-2+w / z}$

Using Eq. (16) the time dependence of the zeroth moment is

$$
\begin{gathered}
\dot{M}_{0} \sim t^{z(\lambda-2)}, \quad \tau<1+\lambda / 2 \\
\sim t^{-2 w}, \quad \tau \geq 1+\lambda / 2 .
\end{gathered}
$$

Equating these time dependences to those of Eq. (14) with $i=0$ and using Eq. (7) we find the additional exponent relations

$$
\begin{aligned}
z & =\frac{1}{1-\lambda}, \quad \tau<1 \\
& =\frac{1}{\tau-\lambda}, \quad 1 \leq \tau<1+\lambda / 2 \\
& =\frac{1}{2-\tau}, \quad \tau \geq 1+\lambda / 2 .
\end{aligned}
$$

These results as well as those for the sum and Brownian kernel are summarized in Table I.

Now we consider the asymptotic regime where the scaling form Eq. (8) is exact. Thus the time derivatives of the moments calculated from Eq. (8) should agree with the time derivatives calculated from the SE, Eq. (16), for all $i$. This requires that we find the time dependence of $I_{i}(K)$ in Eq. (17). First, binomially expand $(x+y)^{i}$ in the integrand for $i \geq 2$. The case for $i=0$ has been worked out above and $i=1$ yields a constant moment. The small- $x$ limit of $\psi(x)$, Eq. (5a), is used to obtain

$$
I_{i}\left(K_{p}\right) \sim \int_{0} d x \int_{0} d y\left[i x^{i-1} y+\frac{i(i-1)}{2} x^{i-2} y^{2}+\cdots+i x y^{i-1}\right](x y)^{\beta-\tau},
$$


TABLE II. Product kernel scaling exponents. Experimental values are the results of the simulations of Kang et al.; theoretical values are the predictions of Table I given the values of $\tau$ from the experiment and the kernel homogeniety, $\lambda$.

\begin{tabular}{|c|c|c|c|c|c|c|c|c|}
\hline & \multicolumn{4}{|c|}{$\lambda=0.50$} & \multicolumn{4}{|c|}{$\lambda=0.80$} \\
\hline & \multicolumn{2}{|c|}{$t \leq 10^{4}$} & \multicolumn{2}{|c|}{$t \geq 10^{4}$} & \multicolumn{2}{|c|}{$t \leq 10^{4}$} & \multicolumn{2}{|c|}{$t \geq 10^{4}$} \\
\hline & Expt. & Theor. & Expt. & Theor. & Expt. & Theor. & Expt. & Theor \\
\hline$w$ & 1.5 & 2.1 & 1.0 & 1.0 & 1.6 & 1.4 & 1.1 & 1.0 \\
\hline$\tau$ & 0.95 & & 1.4 & & 1.3 & & 1.7 & \\
\hline$z$ & 1.4 & 2.0 & 1.9 & 1.7 & 2.4 & 2.0 & 4.0 & 3.3 \\
\hline
\end{tabular}

where use has been made of Eq. (7).

These integrals diverge if $i+\beta-\tau \leq 0$; otherwise, $I_{i}(K)$ is finite and the $t$ dependence of $\bar{M}_{i}$ for $i \geq 2$ and $i+\beta-\tau>0$ is given by

$$
\dot{M}_{i} \sim t^{z(i+\lambda-2)} \text {. }
$$

For the divergent case we transform back to volume space, where the integrand is finite. Upon transformation all the terms in Eq. (23) have the same order in $t$ which is $z(2 \tau-2-\lambda-i)$. Combination into Eq. (16) yields the result

$$
\begin{aligned}
& \dot{M}_{i} \sim t^{z(i+\lambda-2)}, \quad \tau<i+\lambda / 2, \quad i \geq 2 \\
& \dot{M}_{i} \sim t^{-2 w}, \quad \tau \geq i+\lambda / 2, \quad i \geq 2 .
\end{aligned}
$$

For nongelling, mass conservative systems one must have $\tau<2$. Hence, for $\lambda=2 \beta>0$ and $i \geq 2$ both Eq. (14b) and Eq. (24b) are irrelevant. Requiring consistency between $\dot{M}_{i}$ calculated from the scaling distribution, Eq. (14a), and the SE equation, Eq. (24a), we obtain only one condition

$$
z=\frac{1}{1-\lambda}
$$

Since this holds for all $i$, it holds for when the scaling distribution (8) is the correct distribution; hence, Eq. (25) is an asymptotic condition.

We now combine the requirement of moment time evolution consistency for $i \geq 2$, which leads to the wellknown result in Eq. (25), with the similar requirement for $i=0$, which led to the relations in Table I. These conditions should be met in the asymptotic time limit when the scaling distribution is achieved and hence is accurately represented by all its moments. It is evident that the requirement Eq. (25) is met by all three kernels when $\tau<1$. In this case the exponent $w$ is irrelevant because all the moments of the distribution scale as $t^{z(i-1)}$ with $z=1 /(1-\lambda)$. When $\tau>1$, the kernels must be examined separately. With the product kernel, Eq. (25) implies $w=1$ and $\tau=1+\lambda$, in agreement with previously published results. ${ }^{9,10}$ For the sum kernel we only obtain an upper bound on $\tau, \tau \leq 1+\lambda$. This upper limit is also in agreement with previously published results. ${ }^{9}$

\section{COMPARISON WITH COMPUTER SIMULATIONS}

We now compare our results to the simulations of Kang et al. These simulations were performed in the mean-field limit and hence one expects the Smoluchowski equation to be a valid description of the coagulation process. We use their measured value of $\tau$ and the homogeneity $\lambda$ of their coagulation kernels to calculate the exponents $w$ and $z$ using the relations in Table $\mathbf{I}$. The comparisons for the product kernel are summarized in Table II. We note that neither time regime can be considered asymptotic since $\tau$ never equals $1+\lambda$ as it should in the asymptotic regime for the product kernel. Agreement is fairly good since the discrepancies between theory and experiment seen in Table I can be accounted for in all but the short-time $\beta=\lambda / 2=0.25$ values if we assume an error of $5 \%$ or less in the measurements of $\tau$. The discrepancies in the $\beta=0.25$ values can be attributed to the fact that $\tau$ is probably not a weak function of time in this regime, contradicting the original assumption.

The comparisons for the sum kernel are shown in Table III. Excellent agreement is seen for all values of $\beta$. This indicates that $\tau$ is a weak function of time, even early in the simulation and that the zeroth moment, having reached its asymptotic value, provides an accurate description of the size distribution. Thus the time dependence of the exponents is dependent on the form of the coagulation kernel. With the product kernel the exponents reached their asymptotic value relatively quickly, while for the sum kernel they approach relatively

TABLE III. Sum kernel scaling exponents. Experimental values are the results of the simulations of Kang et al.; theoretical values are the predictions of Table I given the values of $\tau$ from the experiment and the kernel homogeneity, $\lambda$.

\begin{tabular}{lcccccr}
\hline & \multicolumn{2}{c}{$\lambda=0.25$} & \multicolumn{2}{c}{$\lambda=0.40$} & \multicolumn{2}{c}{$\lambda=0.75$} \\
& Expt. & Theor. & Expt. & Theor. & Expt. & Theor. \\
\hline$w$ & 1.76 & 1.82 & 1.75 & 1.93 & 2.5 & 2.8 \\
$\tau$ & 0.63 & & 0.84 & & 1.3 & \\
$z$ & 1.32 & 1.33 & 1.56 & 1.67 & 3.3 & 4.0 \\
\hline \hline
\end{tabular}


slowly. This is also manifest in the computer simulations. Kang et al. noted that they were able to reach the asymptotic values of all the exponents using the product kernel but were unable to do with the sum kernel even at the longest time available.

\section{CONCLUSIONS}

The results of Kang et al. suggest that achievement of the asymptotic scaling cluster size distribution and its associated exponents take a considerable amount of time. During this approach to scaling, pseudoscaling is found with nonasymptotic exponents which still obey $w=z(2-\tau)$ if the exponents do not vary too quickly. We can predict the values of these intermediate time exponents by enforcing consistency between the Smoluchowski equation and the scaling distribution ansatz for the zeroth-order moment of the distribution. This implies that during a cluster size distribution's evolution from an arbitrary initial shape to the asymptotic scaling form, the first moment to achieve its asymptotic or scal- ing value is the zeroth moment. When consistency between the SE and the scaling ansatz is enforced for all moments, we found that the usual, asymptotic exponent values were obtained. Hence we propose that higher moments sequentially approach their asymptotic values as the coagulation proceeds.

The consistency-of-moments approach we have used here is simple yet powerful in that it can predict exponents in both the nonasymptotic and asymptotic time regimes if the exponents do not vary too quickly. It would be interesting to simulate the behavior of the moments of a distribution to study how they evolve into their asymptotic values during coagulation.

\section{ACKNOWLEDGMENTS}

We thank B. J. Olivier for helpful discussions. This work was supported in part by the National Science Foundation Grant Nos. CPE-8218415 and CBT-8603736 and a grant from the Research and Creative Activities Committee of the Department of Graduate Studies at Cleveland State University.
${ }^{1}$ Kinetics of Aggregation and Gelation, edited by D. P. Landau and F. Family (North-Holland, Amsterdam, 1984).

${ }^{2}$ On Growth and Form, edited by H. E. Stanley and N. Ostrowski (Nijhoff, Boston, 1986).

${ }^{3}$ Fractals in Physics, edited by L. Pietronero and E. Tosatti (North-Holland, Amsterdam, 1986).

${ }^{4}$ T. Vicsek and F. Family, Phys. Rev. Lett. 52, 1009 (1984).

${ }^{5}$ P. Meakin, T. Vicsek, and F. Family, Phys. Rev. B 31, 564 (1985).
${ }^{6}$ R. L. Drake, in Topics in Current Aerosol Research, edited by G. M. Hidy and J. R. Brock (Pergamon, Oxford, 1972), Vol. 3, Part 2, p. 201.

${ }^{7}$ K. Kang, S. Redner, P. Meakin, and F. Leyvraz, Phys. Rev. A 33, 1171 (1986).

${ }^{8}$ K. W. Lee, J. Colloid Interface Sci. 92, 315 (1983).

${ }^{9}$ P. G. J. van Dongen and M. H. Ernst, Phys. Rev. Lett. 54, 1396 (1985).

${ }^{10}$ F. Leyvraz, Phys. Rev. A 29, 854 (1984). 